\title{
FREQUENCY ANALYSIS OF CARDIAC DATA OBTAINED THROUGH HOLTER MONITORING IN REAL LIVING CONDITIONS
}

\author{
Galya Nikolova Georgieva-Tsaneva ${ }^{1}$
}

\begin{abstract}
The paper presents frequency methods for estimating the variability of intervals between individual heart beats in Electrocardiogram. This parameter is known in the scientific literature as the Heart Rate Variability and with this method it is possible to make predictions about human health. Three frequency ranges have been studied: Very Low Frequency, Low Frequency, and High Frequency. The study in this paper was based on real cardiological data obtained from 33 patients suffering from heart fibrillations and 29 healthy individuals. The investigated records are obtained through a Holter monitoring of studied individuals in real life conditions. The obtained results show significantly lower values of the tested spectral parameters in the diseased individuals compared to the healthy controls. The accomplished study shows the effective applicability of the spectral methods of Heart Rate Variability analysis and the possibility of differentiation by the spectral parameters of the patients from healthy individuals.
\end{abstract}

UDC Classification: 612, DOI: 10.12955/cbup.v7.1498

Keywords: Electrocardiogram, Heart Rate Variability, Frequency domain analysis, heart fibrillation.

\section{Introduction}

Heart rate variability (HRV) is a tool for assessing the time variation of intervals between adjacent cardiac pulsations. HRV is an indirect indicator of the autonomic regulation of the cardiovascular system and it is often used as a prognostic indicator of the health of the human body (Malia et al., 2014). The cardiological intervals change their duration as a result of respiratory activity, blood pressure, physical and mental load, 24-hour rhythms, thermoregulation, etc. Changing the heart rate is a response of the body to the effects of the external environment. As Clifford et al. (2004) write in the time region, the high HRV is a good health indicator, whereas low HRV indicates a high risk of cardiac death resulting from myocardial infarction, heart failure, diabetes, and others. Heart rate is a non-stationary signal and is influenced by the sympathetic and parasympathetic activity of the autonomic nervous system (Acharya et al., 2006). The two parts of the nervous system have different and most often opposite effects on the human regulatory system (Stavrinou, 2014) and they are the main mechanisms of the Heart rate variability (Trimmel, 2015).

The sympathetic nervous system exerts its action in situations of physical exercise (running, wrestling, swimming, sports, etc) and emotional or mental stress, depression where heart rate can rise to 180/200 beats/minute, while increasing power in the LF range. The other part of the autonomic nervous system, the parasympathetic nervous system, acts as a rest, good mood, sleep, cooling, and can reduce heart rate to 60 beats per minute, respectively increasing the power in the HF range. Reducing or increasing the share of the different frequency ranges in the heart rate signal and referencing them to the 24-hour physiological cycle can provide information about the physical and health condition of the person.

The HRV study is based on the measurement of the interval between heart rhythm (RR intervals, where $\mathrm{R}$ is the point with the maximum deviation in the electrocardiogram), and subsequent analysis by various mathematical methods. For this purpose, in the continuous ECG record, the R points are detected, the duration of the intervals between adjacent $\mathrm{R}$ peaks is measured and the instantaneous heart rate is calculated (Georgieva-Tsaneva, 2012). Measurements are based on the intervals between normal sinus abnormalities (occurring as a result of sinus node depolarization) because they reflect the vegetative innervation to the heart ( $\mathrm{NN}$ intervals).

The algorithms for heart rate variability analysis can be divided into three groups (Acharya et al., 2006):

- Algorithms for frequency domain analysis. They give a general analysis of the variability.

- Algorithms for time domain analysis. They are mainly used to obtain statistical estimates.

- Nonlinear algorithms. Through them, specific information on the behavior of the variability can be derived.

\section{Analysis of Variability in the Frequency Area: Literature Review}

In the scientific literature, there are many papers in which the CPS is analyzed, mainly for the influence of various factors such as physical load (Martinis et al., 2004) and stress (Kim et al., 2018; Marques et al., 2010; Dimitriev et al., 2015; Kageyama et al., 1998) and many others.

\footnotetext{
${ }^{1}$ Institute of Robotics, Bulgarian Academy of Sciences, Sofia, Bulgaria, galicaneva@abv.bg
} 
The spectral analysis of the heart rhythm shows the quantitative variation of cyclic effects with a different long cycle over the heart rate. Most of the spectral algorithms apply the window selection method for real-time operation. The following mathematical technologies are most commonly used in frequency areas:

- Discrete Fourier Transform (DFT) and Fast Fourier Transformation (FFT);

- Welch Periodogram;

- Lomb-Scargle Periodogram;

- Method of Burg (Burg Periodogram).

DFT, FFT and the Welch Periodogram are nonparametric methods. In these methods, the spectral analysis is performed directly on the data being studied, and the graph of the spectrum is a series of peaks and falls. In a previous study of the author (Georgieva-Tsaneva, 2016) the method of Welch is used (modification of classical Periodogram) for computing of spectral parameters.

The Lomb-Scargle Periodogram and Burg Periodogram are parametric methods. The parametric methods use an autoregressive model of the data, they do not make spectral analysis of the data itself, but of the obtained model. Thus, the spectrum graph has smooth lines, the spectrum is smoother (Stavrinou, 2014).

Spectral analysis of cardio intervals presents the distribution of frequencies present in NN intervals as a sum of correct sine waves of varying amplitude. A spectral analysis of long-time 24-hour HRV recording in the total frequency range of 0 to $0.5 \mathrm{~Hz}$ distinguishes four sub-bands (according to Hernandez-Fajardo et al., 2008; Georgieva-Tsaneva, 2018), determined for physiological reasons: Ultra Low Frequency (ULF), Very Low Frequency (VLF), Low Frequency (LF) and High Frequency (HF). Following the creation and publication of the common European-American standard in scientific research are using the frequency limits recommended therein (according to Malik, 1996). In the traditional short-term spectral analysis, the VLF, LF and HF areas are examined; at a spectral analysis of long-term records and the ULF range is also investigated. In his paper Trimmel (2015) shows, that good mood and relaxation are correlated with ULF and Total Power. In their research Berntson et al. (1997) point out that the two parts of the nervous system can act not only as antagonists but also in sync in certain situations.

\section{Frequency Domain Analysis}

Of great importance for clinical practice in the study of short-term cardiology series (up to 5-10 minutes) are in the following ranges (Clifford et al., 2006):

- VLF: 0.003-0.04 It reflects the sympathetic activity of the nervous system;

- LF: $0.04-0.15 \mathrm{~Hz}$ with a peak of about $0.1 \mathrm{~Hz}$ over which the sympathetic nervous system affects (Malik, 1996);

- HF: $0.15-0.5 \mathrm{~Hz}$, on which the parasitic portion of the nervous system affects (Malik, 1996), but according to some authors as well the sympathetic nervous system affects (Berntson et al., 1997). HF region reflects the fastest changes in heart rate, with peak peaks around $0.25 \mathrm{~Hz}$.

The relationship between low frequency components and high frequency components (LF / HF) in the HRV spectrum is an important indicator of the cardiological sympathovagal balance in the body (Matveev et al., 2012).

In healthy patients, the LF and HF ranges are changed periodically, with LF influencing prevailing during the day, and $\mathrm{HF}$ at night. For long-term recordings, the low-frequency and high frequency range account for $5 \%$ of the total power spectrum, while the VLF and ULF make up 95\%. Under the influence of various factors, the spectral power of the HF and LF ranges can be increased or decreased, which can be judged for various disorders in the human body. The indicator of cardiological sympathovagal balance can be a predictor of certain diseases.

The frequency spectral parameters for normal HRV (reference values corresponding to healthy individuals) given in HRV standard (according to Malik, 1996) are as follows:

- Total Power: $3466 \pm 1018 \mathrm{~ms}^{2}$;

- LFpower: $1170 \pm 416 \mathrm{~ms}^{2}$;

- HFpower: $975 \pm 203 \mathrm{~ms}^{2}$;

- LF power n.u.: $54 \pm 4$; 
- HFpower n.u.: 29 \pm 3 ;

- LF/HF: 1.5-2.0.

\section{Data used for experimental studies}

The presented studies were performed on patients and healthy subjects - volunteers received by the Medical University - Varna, Republic of Bulgaria, through the Holter monitoring. The received records are over a period of 24 hours, and the studies are performed on a second channel. The used Holter devices are modern, purchased specifically for the purpose of a research project funded by the Research Fund of the Republic of Bulgaria. The main task of the project is to study HRV in patients with various cardiovascular diseases and to monitor their treatment, the effectiveness of the treatment, the results of the assigned medications. The project started with the tracking of patients with heart fibrillations and the analysis of their initial states by means of mathematical methods. The Holter device enables RR intervals to be obtained for each subject being studied, which are recorded in a patient database and can be accessed after a time of comparison and analysis. The analyzed records in this study are for patients aged 25-40 years. The control group of healthy individuals is of the same age group. The records are accompanied with a diagnosis by the investigator doctor, a member of the project team.

\section{Results}

The algorithms that were tested and evaluating their effectiveness were accomplished by means of software for this purpose in a MATLAB environment. All results in the report are obtained with program implementation of the corresponding algorithms.

Obtained results are presented in Table 1 with mean and standard deviation (SD).

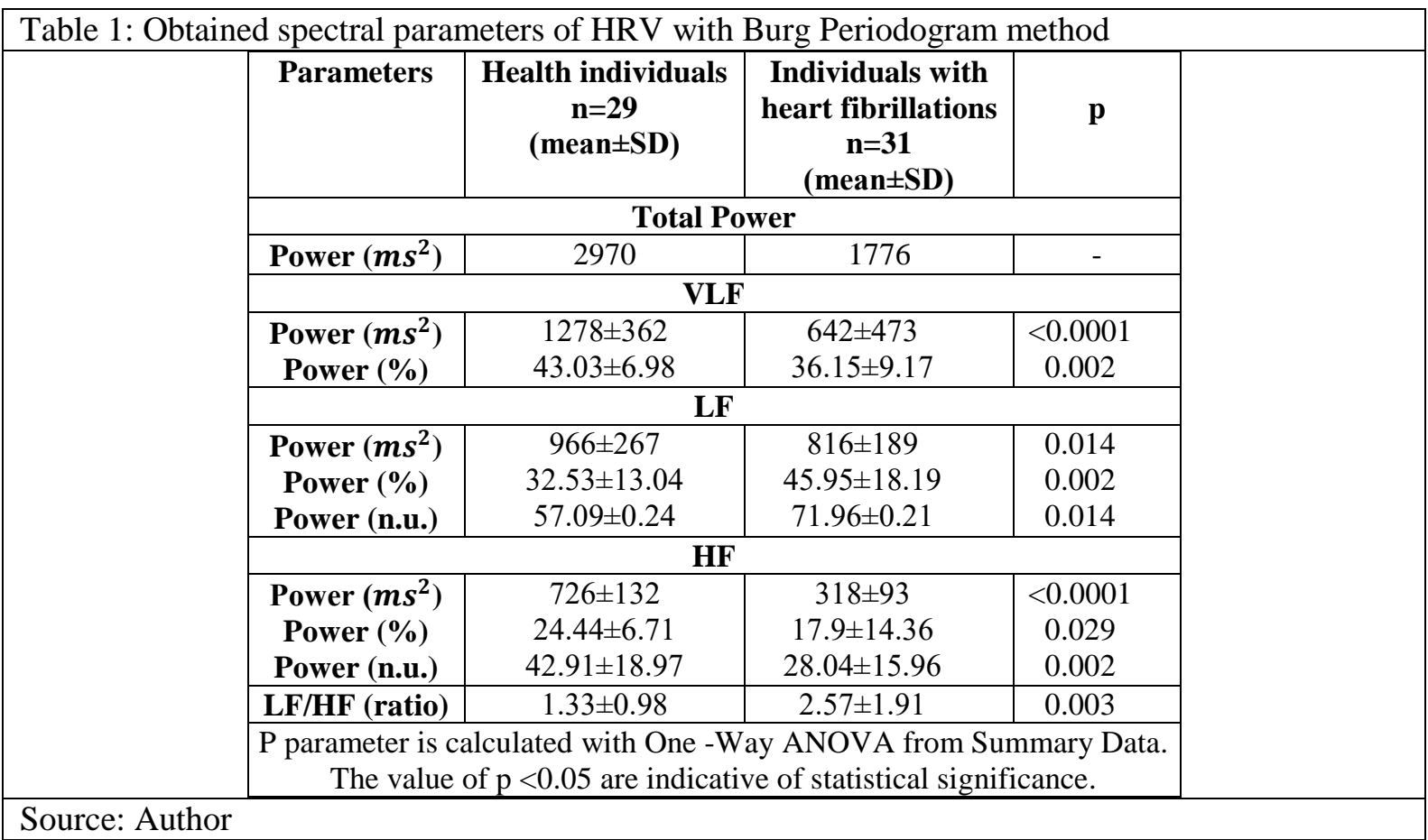

Studies have been done on cardiac records of healthy patients and patients with heart fibrillations. The power of the individual ranges is presented in percentages relative to the total and in normal units. The LF / HF ratio is also calculated, giving information about the sympathovagal balance in the body.

The calculated spectral parameters in the three investigated frequency ranges were obtained by applying a Burg Periodogram method. The results obtained show a decrease in the variability parameters in the VLF, LF and HF range in individuals with heart fibrillations.

The Power in the studied frequency ranges differs in the two study groups of individuals. In the VLF region, the absolute Power in the group with heart fibrillations is $642 \pm 473 \mathrm{~ms}^{2}$ (compared to twice as large $\left(1278 \pm 362 m s^{2}\right)$ in the healthy group). 
The relative share of Power in the LF range (45.95\%) in individuals with heart fibrillations and Power in normal units in relation to the relative share of the LF range and Power in normal units in healthy individuals (32.53\%) are higher. This indicates an increase in sympathetic nervous system activity in patients diagnosed with heart fibrillations, which activity is directed to regulate the imbalance in the diseased human body.

In the HF range is observed a significant decrease in the Power relative share $(17.9 \pm 14.36 \%)$ and in the Power in normal units in patients with heart fibrillations in comparison with the control healthy group (24.44 $\pm 6.71 \%)$. The reducing of the variability in the HF range indicates decreasing the human body's ability to respond quickly to the changing external environment.

Changes in the LF and HF region cause an increase in the sympathetic balance index (LF/HF ratio) in patients with heart fibrillations. The obtained values $(2.57 \pm 1.91)$ for the study group of patients are outside the normal values range (1.5-2) presented in the HRV standard (Malik, 1996) and indicate the presence of health problems.

The value of investigated parameter (calculated with ANOVA analysis) $\mathrm{p}<0.05$ are indicative for statistical significance of obtained results.

Future activity will include monitoring the treatment of the patients under study, respectively at 3 and then every 6 months (if necessary), monitoring the medication used, changing the dose of medications, or switching medicines as needed. The observations made and the outcome of their analysis will be announced at subsequent conferences.

\section{Conclusion}

The report presents a study of Heart Rate Variability in individuals with heart fibrillations, and in healthy individuals. The main aim is to determine whether significant changes occur in HRV in individuals with heart fibrillations. The study was conducted on real cardiac data obtained through a 24-hour Holter monitoring of two groups of individuals: individuals with heart fibrillations and a control group of healthy subjects. A Burg Periodogram method is used to calculate a Periodogram and determine the Power in three frequency ranges: Very Low Frequency, Low Frequency, and High Frequency.

The obtained results show a decrease in HRV in the three frequency ranges examined, indicating that the reduction of $\mathrm{HRV}$ is an indicator of the presence of cardiac disease (in this case heart fibrillations). The presented results indicate a higher relative share of the LF range in patients with heart fibrillations relative to healthy subjects. The low-frequency LF range reflects the influence of the sympathetic part of the nervous system. The increase in power in the LF range is reflected in the LF / HF coefficient, which increases respectively. The results obtained show that lower sympathetic nervous system activity reduces the burden on the heart and contributes to the normalization of its activity.

The statistical analysis done makes it possible to distinguish patients with cardiovascular disease from healthy individuals with HRV Frequency Domain Analysis methods.

The presented results in the paper can be used by cardiologists in their clinical practice as an additional means of determining the patient's disease.

\section{Acknowledgements}

This research was carried out as part of the project "Investigation of the application of new mathematical methods for the analysis of cardiac data" No. KP06-N22/5, 07.12.2018, founded by National Science Found, Bulgaria.

\section{References}

Acharya, U.R., Joseph, K.P., Kannathal, N., Lim, C.M., Suri, J.S. (2006). Heart rate variability: a review. Med. Bio. Eng. Comput, 44, 1031-1051. Retrieved from: https://link.springer.com/article/10.1007\%2Fs11517-006-0119-0, https://doi.org/10.1007/s11517-006-0119-0

Berntson, G.G., Bigger, J.T., Eckberg. D.L., et al. (1997). Heart rate variability: origins, methods and interpretative caveats. Psychophysiology, 34, 623-48. Retrieved from: https://onlinelibrary.wiley.com/doi/abs/10.1111/j.1469-

8986.1997.tb02140.x, https://doi.org/10.1111/j.1469-8986.1997.tb02140.x

Clifford, G., Tarassenko, L. (2004). Segmenting Cardiac-Related Data Using Sleep stages Increases Separation Between Normal Subjects and Apnoeic Patients. IOP Physiol. Means. Retrieved from: https://iopscience.iop.org/article/10.1088/09673334/25/6/N03/meta, https://doi.org/10.1088/0967-3334/25/6/N03

Clifford, G., Azuaje, F., McSharry, P., (Editors), (2006). Advanced Methods and Tools for ECG Data Analysis. Artech House, Inc., Boston/London. 
Hernandez-Fajardo I., Evangelatos G., Kougioumtzoglou I., Ming X. (2008). Signal denoising using wavelet-based methods. Retrieved from: http://cnx.org/content/m18931/1.2/.

Georgieva-Tsaneva, G. (2018). Heart Rate Variability Generating based on Mathematical Tools, Proceedings of the 19th International Conference on Computer Systems and Technologies - CompSysTech'18, (Eds. B. Rachev, A. Smrikarov), ACM New York, NY, USA, 134-138, https://doi.org/10.1145/3274005.3274035

Georgieva-Tsaneva, G. (2016). Application of Mathematical Methods for Analysis of Digital ECG Data, Information Technologies, and Control, 2, 35-43. Retrieved from: http://archive.sciendo.com/ITC/itc.2016.14.issue-2/itc-2017-0005/itc2017-0005.pdf, https://doi.org/10.1515/itc-2017-0005

Georgieva-Tsaneva, G. (2012). QRS detection algorithm for long term Holter records. Proceedings of 14th International Conference on Computer Systems and Technologies-CompSysTech'13 (Eds. B. Rachev, A. Smrikarov), ACM New York, NY, USA, 112-119, https://doi.org/10.1145/2516775.2516811

Dimitriev, D.A., Saperova, E.V. (2015). Heart rate variability and blood pressure during mental stress. Rossiiskii Fiziologicheskii Zhurnal Imeni I. M. Sechenova,101, 98-107.

Kageyama, T., Nishikido, N., Kobayashi, T., Kurokawa, Y., Kaneko, T., Kabuto, M. (1998). Self-reported sleep quality, job stress, and daytime autonomic activities assessed in terms of short-term heart rate variability among male white-collar workers. Industrial Health, 36, 263-272. Retrieved from:

https://www.jstage.jst.go.jp/article/indhealth1963/36/3/36_3_263/_article, https://doi.org/10.2486/indhealth.36.263

Kim, H., Cheon E., Bai, D., Lee, Y., Koo, B. (2018). Stress and Heart Rate Variability: A Meta-Analysis and Review of the Literature. Psychiatry investigation, 15(3), 235-245. Retrieved from: https://www.ncbi.nlm.nih.gov/pmc/articles/PMC5900369/, https://dx.doi.org/10.30773\%2Fpi.2017.08.17

Mali, B., Zulj S., R. Magjarevic, D. Miklavcic, T. Jarma. (2014). Matlab-based tool for ECG and HRV analysis. Biomedical Signal Processing and Control, 10, 108-116. Retrieved from: http://lbk.electroporation.net/pdfs/bspc2014.pdf, http://dx.doi.org/10.1016/j.bspc.2014.01.011

Malik, M. (1996). Task Force of the European Society of Cardiology and the North American Society of Pacing and Electrophysiology. Heart Rate Variability: Standards of Measurement, Physiological Interpretation, and Clinical Use. Circulation, 93, 1996, 1043-1065. Retrieved from: https://www.ncbi.nlm.nih.gov/pubmed/8598068, https://doi.org/10.1161/01.CIR.93.5.1043

Martinis M., Knezevic A., Krstacic G., Vargovic E. (2004). Changes in the Hurst exponent of heart beat intervals during physical activities. Physics Rev E 70:012903. Retrieved from:

https://journals.aps.org/pre/abstract/10.1103/PhysRevE.70.012903, https://doi.org/10.1103/PhysRevE.70.012903.

Marques A.H., Silverman M.N., Sternberg E.M. (2010). Evaluation of stress systems by applying noninvasive methodologies: measurements of neuroimmune biomarkers in the sweat, heart rate variability and salivary cortisol. Neuroimmunomodulation, 17, 205-208. Retrieved from:

https://www.ncbi.nlm.nih.gov/pubmed/20134204, https://doi.org/10.1159/000258725.

Matveev M., Krasteva V., Jekova I., Georgiev G., Milanov St., Prokopova R., Todorova L. (2012). Profile of Autonomic Cardiac Control in Patients who are Not Considered Ready for Weaning from Mechanical Ventilation. Computing in Cardiology, 39, 625-628. Retrieved from: https://ieeexplore.ieee.org/document/6420471

Stavrinoua, M, Sakellaropoulos G., Trachani F., Sirrouc V., Polychronopoulos P., Nikiforidis G., Chroni E. (2014). Methodological issues in the spectral analysis of the heart rate variability: Application in patients with epilepsy. Biomedical Signal Processing and Control, 13, 1-7. Retrieved from:

http://www.sakellaropoulos.gr/Publications/J69_Biom_Sign_Proc_Control_2014_13_1-7.pdf, http://dx.doi.org/10.1016/j.bspc.2014.03.002.

Trimmel, M. (2015). Relationship of Heart Rate Variability (HRV) Parameters Including pNNxx With the Subjective Experience of Stress, Depression, Well-Being, and Every-Day Trait Moods (TRIM-T): A Pilot Study. The Ergonomics Open Journal, 2015, 8, 32-37. Retrieved from:

https://pdfs.semanticscholar.org/dc38/50a5f44b84193fa62485091463eae04a0bfd.pdf, http://dx.doi.org/10.2174/1875934301508010032. 